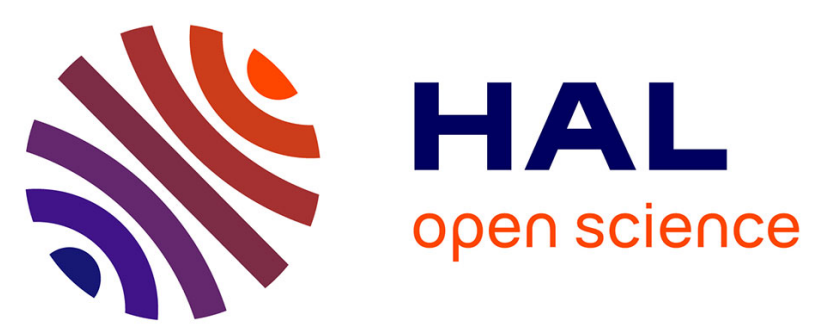

\title{
"Hypocrite lecteur, mon semblable, mon frère!" : le roman populaire du XIXe siècle et de la Belle Époque entre manichéisme et mauvaise foi
}

Jacques Migozzi

\section{- To cite this version: \\ Jacques Migozzi. "Hypocrite lecteur, mon semblable, mon frère! ": le roman populaire du XIXe siècle et de la Belle Époque entre manichéisme et mauvaise foi. Flocel Sabaté. Perverse identities. Identities in conflict, Peter Lang, 2015. hal-02437764}

\section{HAL Id: hal-02437764 \\ https://hal-unilim.archives-ouvertes.fr/hal-02437764}

Submitted on 13 Jan 2020

HAL is a multi-disciplinary open access archive for the deposit and dissemination of scientific research documents, whether they are published or not. The documents may come from teaching and research institutions in France or abroad, or from public or private research centers.
L'archive ouverte pluridisciplinaire HAL, est destinée au dépôt et à la diffusion de documents scientifiques de niveau recherche, publiés ou non, émanant des établissements d'enseignement et de recherche français ou étrangers, des laboratoires publics ou privés. 


\title{
« Hypocrite lecteur, mon semblable, mon frère ! » : le roman populaire du XIXe siècle et de la Belle Époque entre manichéisme et mauvaise
}

\section{foi.}

\author{
Jacques Migozzi \\ Université de Limoges
}

« Le génie du mal a recruté une armée de poètes et de romanciers dont le talent a contribué plus qu'on ne le pense à l'explosion des révolutions qui sont venues périodiquement affliger la France. [...] Nous considérons comme perdus pour la double cité du ciel et de la terre ceux qui lisent certains romans que tout le monde désigne... » Comme en témoigne ce prospectus alarmiste diffusé en 1853 par les éditeurs de la Bibliothèque des poètes et romanciers chrétiens, stigmatisant le roman populaire et son influence éminemment vénéneuse, les fictions de grande consommation ont été dès la « querelle du roman-feuilleton » 1 et l'avènement de « la civilisation du journal »2, autrement dit dès l'entrée de la littérature dans l'ère médiatique et de la culture-marchandise, accusées de pervertir les lecteurs (et surtout les lectrices !) sous couvert de les divertir. Ce discours véhément de prophylaxie sociale et morale, dont l'abbé Bétlhéem se fera une spécialité au début du XXème siècle avec sa célèbre sélection commentée Romans à lire et à proscrire, sera repris inlassablement par les élites de tous bords jusqu'à la fin du XXe siècle, les progressistes laïques puis les marxistes s'entendant au moins sur un point avec les chrétiens les plus intransigeants 3 : le roman populaire est aliénant car il égare et mystifie ; le roman populaire est dangereux car il flatte la « folle du logis » et stimule les

\footnotetext{
1 Lise Dumasy, La querelle du roman-feuilleton - Littérature, presse et politique, un débat précurseur (1836-1848) (Grenoble: ELLUG, Université Stendhal, 1999).

2 Dominique Kalifa, Philippe Régnier, Marie-Eve Thérenty, Alain Vaillant, La Civilisation du Journal. Histoire culturelle et littéraire de la presse française au XIXème siècle (Paris : Nouveau monde éditions, 2012).

3 «De nos jours, la peste est inoculée aux âmes à profusion sur nos marchés littéraires et le peuple, alléché par l'attrait de ce poison, l'achète en quantité ; il lit avec avidité des romans qui doivent leur succès à leur caractère pornographique et à la glorification des instincts les plus bas. On sait, hélas! les conséquences. Opposer à cette épouvantable intoxication, qui éteint tout sentiment élevé et tue les âmes, des lectures saines, intéressantes, populaires, à des prix infimes, voilà quel serait le sérum opportun. » (Prospectus de 1912 des éditions de la Bonne Presse pour la présentation de «Stella », nouvelle collection du Petit Echo de la mode).
} 
fantasmes, donc favorise l'émergence d'une identité perverse chez le lecteur pris dans les rets du romanesque.

Ces mêmes récits plébiscités par le grand public ont été en revanche ultérieurement épinglés pour leur manichéisme, dispensant en même temps que «l'espoir et la consolation » un prêt-à-penser / prêt-à-rêver fondé sur une vision archaïque : conséquemment, dans le roman populaire riche en péripéties, par delà le ballet des déguisements, des quêtes ou usurpations d'identité, les bons sont bons, les méchants sont méchants, autrement dit le lecteur ne devrait pas être troublé par l'identité perverse de personnages ambigus mais au contraire conforté dans son axiologie par le dévoilement de l'identité véritable, quand tombent les masques et que les identités temporairement perverties - donc, conformément à l'étymologie du latin pervertere, mises sens dessus dessous - se retrouvent au dénouement rétablies. Stricto sensu, l'identité des personnages dans le roman populaire du XIXe siècle et de la Belle Epoque n'apparaîtrait donc pas perverse en dernière instance, même si fréquemment des suborneurs ou des infâmes, voire des pervers, s'emploient à pervertir les identités avant d'être châtiés. (Qu'il soit clair que mon propos ne raisonne que sur ce corpus, et que les hypothèses ici présentées, si elles demeurent probablement valables pour une large part pour les fictions populaires multimédiatiques ultérieures, devraient être fortement nuancées dès lors qu'on s'attacherait à des récits plus récents, en particulier par exemple aux séries télévisées contemporaines construites autour de héros ambivalents : Desperate housewives, Dexter, Mad men, Doctor House...)

En me fondant sur les travaux de quelques illustres prédécesseurs ou partenaires de recherche au long cours, pionniers dans le défrichement du roman populaire et de ses genres (Jean-Claude Vareille, Umberto Eco, Daniel Couégnas, Paul Bleton, Matthieu Letourneux) et en y mêlant quelques considérations de mon cru, je tenterais aujourd'hui à gros traits de rendre raison de ce paradoxe, selon lequel le récit paralittéraire pourrait pervertir le lecteur lors même qu'il déploierait un « système « pansémique », redondant, marqué par la polarisation idéologique » (critère $3 \mathrm{du}$ 《 modèle paralittéraire » proposé par Couégnas dans son ouvrage fondamental4). Pour ce faire, je prendrai pour fil directeur de mon propos la représentation des figures criminelles dans le roman populaire français de 1840 à 1914 et leur recours à la dissimulation de leur identité, avec pour terminus la spectaculaire floraison au début du XXe siècle de la cohorte prestigieuse des « voleurs de 
visages »5 tels qu'Arsène Lupin, Chéri Bibi et surtout Fantômas, « l'Insaisissable, le Maître de l'effroi ». Avec ce dernier « génie du crime » et la mascarade définitive qu'il impose sur l'identité, on pourra peut-être alors saisir emblématiquement que la séduction du jeu romanesque tient au miroir que tend cette «feintise ludique partagée » 6 aux fantasmes du lecteur.

Comme l'a souvent martelé avec brio Jean-Claude Vareille, le roman populaire (français) du grand Dix-Neuvième siècle - et probablement bon nombre de ses avatars postérieurs - déploie des représentations expressionnistes irriguées par une Vision des Essences, nourrie comme dans les sociétés dites primitives, d'une rêverie somatique et ontologique. Plus que des caractères subtilement travaillés, les figures criminelles incarnent du même coup des types à tendance allégorique : le Traitre (de mélodrame !), la Femme fatale, l'Espion, le Suborneur, l'Ogre ou l'Ogresse (c'est le cas de le dire dans Les Mystères de Paris !)..., tous pourvus d'une identité stable et avant tout fonctionnelle. Comme le dit en effet avec justesse Daniel Couégnas : «Le «Méchant : sa fonction narrative est de créer le scandale. Sa fonction pragmatique est d'indigner et/ou de faire peur »7, ce qu'emblématise avec une géniale malice en février 1911 le célèbre incipit de la série de 32 volumes des Fantômas publiés en 32 mois par Pierre Souvestre et Marcel Allain dans la collection du Livre Populaire d'Arthème Fayard :

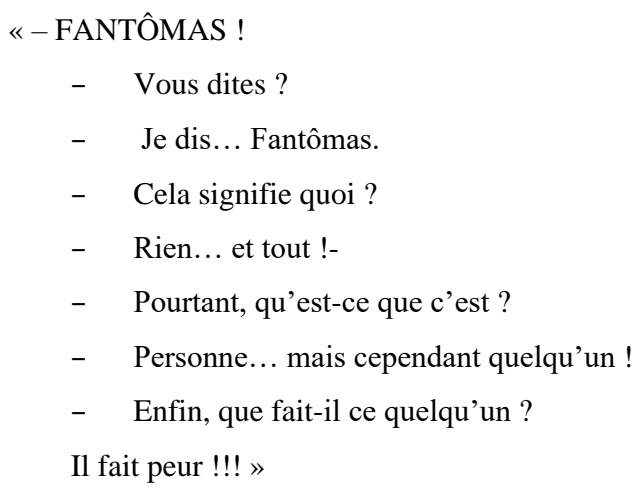

Selon la position systémique qui leur est assignée dans un dispositif manichéen - comme dans la distribution des rôles narratifs tout au long de la série des James Bond de Ian

5 Didier Blonde, Les voleurs de visage, (Paris: Editions A. M. Métailié, 1992).

6 Jean-Marie Schaeffer, Pourquoi la fiction? (Paris: Seuil, 1999).

7 Daniel Couégnas, Introduction à la Paralittérature..., p 173. 
Fleming, telle que 1' a décryptée Umberto Eco dans une analyse désormais classique 8 les Méchants sont donc tout d'une pièce, leur identité est fort rarement clivée. Certes, certains infâmes connaissent leur rédemption, mais il s'agit alors d'une conversion irréversible, jamais d'une oscillation axiologique qui pourrait les rendre pervers : abandonné défiguré et pantelant au bagne de Toulon, le monstrueux Rocambole des premiers volumes des Drames de Paris de Ponson du Terrail ressurgit métamorphosé physiquement et moralement dans La[bien nommée !] Résurrection de Rocambole en 1866 pour traquer désormais les criminels et jouer le Justicier, protecteur de la veuve et de l'orphelin, avec l'appui de l'ancienne prostituée Baccarat, devenue elle-même sœur Marianne.

Cet « imaginaire sursaturé de valeurs », « où l'éthique, l'esthétique et l'ontologique, en position de réversibilité et d'implication réciproque ne font en définitive qu'un $\gg 9$ hérite incontestablement, lorsqu'il s'agit de donner corps aux criminels, des codes imposés dès le XVIIIe siècle par la physiognomonie de Lavater, que la caricature a contribué à disséminer tout au long du XIXe siècle, et dont la vogue de l'anthropologie criminelle de Cesare Lombroso propose à la fin du siècle une version positiviste et rationalisée. En bref, le Méchant a le physique de son emploi. Version noble : le prince de Gonzague a la beauté du Diable, androgyne, italienne et noire, à l'image de sa scélératesse de grand seigneur déviant :

«Gonzague était un homme de trente ans, un peu efféminé de visage, mais d'une beauté rare au demeurant. Impossible de trouver plus noble tournure que la sienne. Ses cheveux noirs, soyeux et brillants, s'enflaient autour de son front plus blanc qu'un front de femme [...] Ses yeux noirs avaient le regard clair et orgueilleux des gens d'Italie. Il était grand, merveilleusement taillé ; sa démarche et ses gestes avaient une majesté théâtrale. »10

A opposer bien sûr à l'harmonie toute française, virile et blonde du chevalier de Lagardère :

« Henri de Lagardère était d'une taille un peu au-dessus de la moyenne. Ce n'était pas un Hercule ; mais ses membres avaient cette vigueur souple et gracieuse du type parisien [...] Il avait des cheveux blonds, légèrement bouclés, plantés haut et découvrant un front

\footnotetext{
8 Umberto Eco, «Structures narratives chez Fleming », De Superman au Surhomme (Paris: Grasset, 1993) [Il superuomo di massa, Milano : Bompiani, 1978 ].

9 Jean-Claude Vareille, «Les images d'Épinal sont-elles édifiantes? », L'édification. Morales et cultures au XIXe siècle, Stéphane Michaud, dir. (Paris: Créaphis, 1993), p 97.

10 Paul Féval, Le Bossu (Paris: GF Flammarion, 1997), p 53.
} 
qui respirait l'intelligence et la noblesse. [...] Le sourire du gai vivant n'effaçait point la fierté du porteur d'épée. Mais ce qui ne se peut peindre à la plume, c'est l'attrait, la grâce, la juvénile gaillardise de cet ensemble. »11

Version canaille, où l'infamie donne dans la difformité : voici l'entrée en texte dans le chapitre 5 des fondateurs Mystères de Paris, du Chourineur, tout à la fois léonin et simiesque, et de sa «gerce», la Chouette, dont le nom parle de lui-même. C'est un véritable feu d'artifice du bestiaire lavaterien :

«Une sorte de frémissement de terreur courut parmi les hôtes du tapis-franc.

Rodolphe lui-même, malgré son intrépidité naturelle, ne put vaincre une légère émotion à la vue de ce redoutable brigand, qu'il contempla pendant quelques instants avec une curiosité d'horreur. [...]

On ne pouvait voir quelque chose de plus épouvantable que le visage de ce brigand. Sa figure était sillonnée en tous sens de cicatrices profondes, livides; l'action corrosive du vitriol avait boursouflé ses lèvres ; les cartilages du nez ayant été coupés, deux trous difformes remplaçaient les narines. Ses yeux gris, très clairs, très petits, très ronds, étincelaient de férocité ; son front, aplati comme celui d'un tigre, disparaissait à demi sous une casquette de fourrure à longs poils fauves ... on eût dit la crinière d'un monstre. Le Maître d'école n'avait guère plus de cinq pieds deux ou trois pouces; sa tête, démesurément grosse, était enfoncée entre ses deux épaules larges, élevées, puissantes, charnues, qui se dessinaient sous les plis flottants de sa blouse de toile écrue; il avait les bras longs, musculeux; les mains courtes, grosses et velues jusqu'à l'extrémité des doigts ; ses jambes étaient un peu arquées, mais leurs mollets énormes annonçaient une force athlétique.

Ce homme offrait, en un mot, l'exagération de ce qu'il y a de court, de trapu, de ramassé dans le type d'Hercule Farnèse.

Quant à l'expression de férocité qui éclatait sur ce masque affreux, quant à ce regard inquiet, mobile, ardent comme celui d'une bête sauvage, il faut renoncer à les peindre.

[...] Rodolphe la voyait de profil ; son œil vert et rond, son nez crochu, ses lèvres minces, son menton saillant, sa physionomie à la fois méchante et rusée, lui rappelèrent la Chouette $\gg 12$

11 Paul Féval, Le Bossu ..., p 97.

12 Eugène Sue, Les Mystères de Paris, (Paris: Albin Michel/Hallier, 1977), p. 48 [Première partie, chapitre 5]. 
Plus d'un siècle plus tard, Ian Fleming activera les mêmes clichés en dotant la cohorte des adversaires de 007 des mêmes attributs monstrueux comme le soulignera Umberto Eco13 : cheveux roux, en apanage démoniaque du Chiffre (Casino Royal), de Hugo Drax (Entourloupe dans l'azimut), de Goldfinger, de Rosa Klebb (Bons baisers de Russie), Jack Spang (Les diamants sont éternels); mains velues et tête énorme (Hugo Drax) ou absence de cou (Jack Spang, Rosa Klebb, Goldfinger)14. Ainsi guidé sur le plan herméneutique par cette pansémie de signes redondants, le lecteur à plus d'un titre averti du roman populaire n'est jamais dupe quant à l'identité des méchants, fût-elle dissimulée. Au besoin le texte n'hésite guère à l'alerter ou plutôt à le conforter dans son diagnostic, comme dans La Porteuse de pain de Xavier de Montépin (1884) lorsque l'assassin Jacques Garaud s'affiche sous une identité usurpée, celle de Paul Harmant, nom d'emprunt qui apparaît en signature d'une lettre :

«Dans ce voyageur nos lecteurs ont reconnu déjà, malgré sa transformation et le changement de couleur de sa chevelure, Jacques Garaud, le contremaître de la fabrique d'Alfortville; Jacques Garaud, l'incendiaire; Jacques Garaud, l'assassin de son patron. »15

Si le lecteur connaît donc d'emblée les méchants, il peut aussi approuver les Bons contraints parfois, mais rarement, par une nécessité éminemment supérieure à assumer en apparence une identité perverse, celle de l'espion. Paul Bleton, dans son ouvrage La cristallisation de l'ombre. Les origines oubliées du roman d'espionnage sous la IIIe République cite ainsi un monologue de la vaillante Germaine Aubry, dans Coeur de Française d'Arthur Bernède, (Tallandier, Le Livre national (série rouge), 1919) :

«Ai-je bien le droit de faire cela ? [...] Va, courage !... tu n'es pas l'espionne qui trahit et qui ment ... Tu es le soldat envoyé en reconnaissance et qui cherche à surprendre l'ennemi... Tu es l'extrême avant-garde d'une armée qui attend de toi les renseignements qui vont éclairer sa route ! ... En avant Française !... En avant toujours et quand même !» et le commente judicieusement :

\footnotetext{
13 Umberto Eco, « Structures narratives chez Fleming »..., pp. 196-200.

14 « Cette singulière unité physionomique des Méchants de service confère une certaine unité au rapport Bond-Méchant, d'autant que le Méchant se distingue également par une série de caractéristiques raciales et biographiques ». Voir: Umberto Eco, « Structures narratives chez Fleming »..., pp. 200-201.

15 Xavier de Montépin, La Porteuse de pain (Paris: Presses de la Cité, coll. Omnibus, 1992), p. 73.
} 
«C'est le pathos patriotique qui est chargé de résoudre la contradiction entre éthique et espionnage, d'inventer une morale patriotique supérieure à la morale naturelle : la duplicité s'en voit en même temps assumée et effacée. »16 Bref, lorsque surgit lors des « lectures dérobées » du « roman du quotidien »17 le tandem des Affreux et des Justes le lecteur est en pays de connaissance, d'autant que cette vision des Essences est propagée de manière hyperbolique et constante par la presse à grand tirage à compter des années 1880, qui spectacularise le fait divers en construisant médiatiquement un imaginaire du crime, comme l'ont souligné les travaux des historiens Anne-Claude Ambroise-Rendu ou Dominique Kalifa. Mais ce fixisme ontologique, qui rassure tout autant qu'il effraie, ne dissimule-t-il pas un questionnement taraudant, qui hante la Fin de siècle, sur l'identité énigmatique des individus et la part d'ombre que recèle chacun? Les méchants stéréotypés du roman populaire sont après tout contemporains des névrosés de la décadence et des premiers travaux de Freud... C'est donc fort probablement à une hantise sociétale sur l'identité et ses abysses qu'il convient de rapporter la floraison dans les premières années du XXe siècle de toute une phalange de bandits masqués et voleurs de visages, et ce de manière transnationale et transmédiatique : Raffles, Arsène Lupin, Lord Lister, Zigomar, Chéri-Bibi, Fantômas...

Bondissant des feuilletons, fascicules, dime novels ou romans bon marché vers les écrans du muet sous la forme de serials, les gentlemen-cambrioleurs et les génies du crime semblent de fait se disputer la vedette dans les années précédant la Grande guerre 18. Tous sont des descendants de Protée, virtuoses dans l'art du déguisement, insaisissables. Pourtant, si tous fascinent le grand public, leur charme n'est pas de même nature. Plus séducteur que menaçant, le gentleman-cambrioleur continue à assumer, par delà son panache de hors-la-loi, l'héritage gratifiant du héros épique, à l'occasion justicier libertaire. Si son identité est dissimulée à plaisir, le jeu de masques ne vaut donc pas symptôme d'une identité perverse du protagoniste, et si dès sa première apparition en 1905 dans la nouvelle «L'arrestation d'Arsène Lupin », le héros de Maurice Leblanc se montre pervers ce n'est pas en tant que personnage mais bien en tant que narrateur.

\footnotetext{
16 Paul Bleton, La cristallisation de l'ombre. Les origines oubliées du roman d'espionnage sous la IIIe République (Limoges: PULIM, Médiatextes, 2011), p 151.

17 Anne-Marie Thiesse, Le Roman du Quotidien (Paris: Points Seuil, 2000).

18 Voir le musée virtuel : «Popular Roots of European Culture» <http://www.popular-roots.eu>; et les expositions thématiques « Fantômas et l'Européenne du crime » <http://fantomas.popular-roots.eu $\rangle$ et « Gentleman-cambrioleur » $<$ http://gentleman.popular-roots.eu>
} 
Trompé savoureusement par le texte, comme il le sera à l'identique 21 ans plus tard par Agatha Christie dans Le Meurtre de Roger Ackroyd, le lecteur entend d'abord en effet le panégyrique de Lupin par un «je » qui se fond dans la communauté des passagers du transatlantique :

«Arsène Lupin parmi nous ! l'insaisissable cambrioleur dont on racontait les prouesses dans tous les journaux depuis des mois ! l'énigmatique personnage avec qui le vieux Ganimard, notre meilleur policier, avait engagé ce duel à mort dont les péripéties se déroulaient de façon si pittoresque! [...] Arsène Lupin, l'homme aux mille déguisements : tour à tour chauffeur, ténor, bookmaker, fils de famille, adolescent, vieillard, commis voyageur marseillais, médecin russe, torero espagnol !», mais c'est pour découvrir trois pages avant la fin du récit que le narrateur d'Andresy n'était autre que Lupin ! 19 Avec rouerie et élégance, le gentleman-cambrioleur nous convie donc à jouer avec les apparences, sans coup férir.

Avec Fantômas, « Maître de l'épouvante », il n'en va plus de même : le jeu de masques terrifie au lieu de divertir, et génère un vacillement inquiétant de l'identité. On se permettra donc ici, après d'autres, et notamment Jean-Claude Vareille, de poser le démoniaque héros de Souvestre et Allain comme un terminus ad quem de cette bande de héros criminels peuplant le roman populaire du XIXe siècle et de la Belle Epoque.

Véritable mythe moderne, et recensé comme tel dans le Dictionnaire des mythes fantastiques de Pierre Brunel et Juliette Vion-Dury20, Fantômas subjugue le grand public dès son apparition en février 1911 dans le premier des 32 volumes, écrits/dictés « à la vapeur » et à quatre mains par Souvestre et Allain entre février 1911 et septembre 1913). Son aura maléfique l'érige en allégorie du crime, comme le rappelle l'ultime opus de la série initiale, La Fin de Fantômas.

«Fantômas n'était pas, en effet, seulement, le Roi de l'épouvante, le Maître de l'effroi, le Génie du crime, le Tortionnaire inégalable, c'était surtout l'Insaisissable.

Fantômas, c'était le légendaire bandit que tous les services de la Sûreté s'efforçaient d'atteindre depuis des années, et que l'on n'arrivait jamais à appréhender. »21

19 Maurice Leblanc, «L'arrestation d'Arsène Lupin », in Arsène Lupin gentleman-cambrioleur, (Paris: Pierre Lafitte, 1907), pp. 13-14.

20 Pierre Brunel, Juliette Vion-Dury, Dictionnaire des mythes du fantastique (Limoges: PULIM, 2003).

21 Alain Souvestre, Marcel Allain, « La Fin de Fantômas », Fantômas (Paris: Robert Laffont, 1988), p. 823. 
Fantômas, c'est donc avant tout un « nom lugubre, [un] nom d'horreur, [un] nom de sang, [un] nom de mort », qui suscite le (délicieux) frisson de la terreur par sa seule évocation sous forme de cri et le charme (littéralement ) du signifiant. Plus rarement, car on parle/fantasme sur Fantômas bien plus souvent dans le récit qu'on ne l'aperçoit, ce héros criminel se résume à une «silhouette d'horreur, silhouette d'épouvante, silhouette maudite ! ...»22:

«Son aspect était fantastique, et le malheureux Jules trembla de tous ses membres...

[...] Il était des pieds à la tête, moulé dans une sorte de tricot noir, semblable à ceux qu'avaient adoptés depuis longtemps les rats d'hôtel, vêtements sinistres qui permettent à ceux qui les portent, de se confondre avec la nuit, d'être à peu près insoupçonnables dans un endroit sombre, alors que tout autre habillement, fatalement, fait un peu tache de lumière et risque d'attirer les regards ...

Mieux que les rats d'hôtel, d'ailleurs, l'homme avait soigné son costume !

Il n'avait pas, en effet, le visage à découvert ; celui-ci était entièrement dissimulé sous une longue cagoule noire, masque flottant, qui empêchait de voir ses traits et où s'allumaient seulement, comme deux charbons ardents, les reflets de ses deux prunelles... $\gg 23$

Par delà cette magnétique effigie de criminel masqué, promise à une expansion iconique quasi inépuisable dans «la mémoire rétinienne » (Pierre Nora) du grand public via le cinéma et la bande dessinée, qui est toutefois Fantômas ? C'est justement là le problème pour tous ceux qui le traquent ou tentent de l'approcher, de ses increvables adversaires diégétiques Juve et Fandor jusqu'aux lecteurs d'hier et d'aujourd'hui !

«Car, qui est Fantômas en tant que Fantômas ? peux-tu bien me le dire, Fandor? poursuivit Juve qui commençait à s'animer.

Certes, nous avons vu au cours de notre existence mouvementée un vieux monsieur comme Etienne Rambert, un anglais trapu comme Gurn, un robuste gaillard comme Loupart, un chancelant et maladif individu comme Chaleck. Nous les avons reconnus tour à tour pour être Fantômas et c'est tout.

Quant à voir Fantômas lui-même, tel qu'il est, sans artifice, sans fard, sans barbe postiche, sans perruque mobile, Fantômas, tel que son visage est sous sa cagoule noire....voilà ce

22 Alain Souvestre, Marcel Allain, « La Fin de Fantômas »..., p. 892-893.

23 Pierre Souvestre, Marcel Allain, Fantômas. Le mort qui tue (Paris: Fayard, 1911), p. 188. 
que nous n'avons pas encore obtenu, réalisé ; voilà ce qui rend notre chasse au bandit sans cesse difficile, souvent périlleuse ...

Fantômas est toujours quelqu'un, parfois deux personnages, jamais lui-même ! » 24

Dans la France du début du XXe siècle, au moment où se développent avec l'anthropométrie, le relevé des marques corporelles, la photographie signalétique... les premières tentatives systématiques de contrôle des individus par l'Etat, Fantômas seraitil dans ces conditions un symbole de résistance à une surveillance généralisée ? Ce qui est certain en tous cas, c'est que Juve et Fandor fréquentent l'inventeur de ces méthodes, le fameux Alphonse Bertillon, mais que les mesures scientifiques de ce dernier sont déjouées par Fantômas, qui va jusqu'à se fabriquer des gants en peau humaine pour égarer les enquêteurs modernistes adeptes des empreintes digitales! Par delà ce potentiel libertaire dont certains ont pu créditer l'Insaisissable, Jean-Claude Vareille voit toutefois avec raison dans son identité perpétuellement évanescente le fondement d' une force dérangeante inédite :

«Et ici éclate la modernité du personnage comme du texte qui le supporte : il n'y a pas chez Fantômas d'être d' au-delà de l'apparence, l'apparence est son être. Fantômas n'a qu'une essence: son masque. On peut donc contempler seulement ses avatars successifs $\gg 25$

Amplifiant son propos, il s'avance alors vers des conclusions qui nous concernent au premier chef :

« [...] le cycle de Fantômas, comme celui de Chéri-Bibi son contemporain, appartient à l'esthétique baroque, celui du reflet et du faux-semblant, du dédoublement et du morcellement irrépressible des apparences. La logique aristotélicienne de l'identité, de la non-contradiction et du tiers-exclu vacille; les contraires fusionnent, l'unité se scinde, les surfaces et les êtres se mettent à briller et à se renvoyer des images tournoyantes : nous sommes au centre d'un labyrinthe de foire où les glaces ménagent de fausses sorties et rendent palpables d'invisibles obstacles.»26

Fantômas ne se contente pas en effet d'être masqué, son essentielle (littéralement) hypocrisie (là encore littéralement)identitaire fait de lui une enveloppe qui dissimule un vide vertigineux, car avec lui la sarabande carnavalesque des jeux de masque n' jamais

\footnotetext{
24 Pierre Souvestre, Marcel Allain, Fantômas. La Main coupée (Paris: Fayard, 1911), p. 241.

25 Jean-Claude Vareille, L'Homme Masqué, le Justicier et le Détective (Lyon: Presses Universitaires de Lyon, 1989), p. 144.

26 Jean-Claude Vareille, L'Homme Masqué, le Justicier et le Détective..., p 139.
} 
de fin. «Dans Le Policier apache27, cette technique du miroitement atteint un paroxysme difficilement égalable » dans le chapitre XVIII« Fantômas contre Fantômas » : lors d'un bal masqué organisé chez la grande-duchesse Alexandra, alias Lady Beltham, maîtresse du Génie du crime, ce ne sont pas moins de plusieurs Fantômas qui se croisent et dialoguent, sans qu'on puisse toujours savoir si celui qui parle est Jérôme Fandor déguisé en Fantômas, Tom Bob, le célèbre détective américain, lui aussi déguisé en Fantômas, un policier de surveillance déguisé en Fantômas et qui finira assassiné, ou Fantômas luimême déguisé en ... Fantômas. Il est vrai qu'on apprendra deux cents pages plus loin que Tom Bob avait depuis belle lurette été assassiné et que Fantômas avait usurpé son identité, ce qui permet d'ailleurs à Tom Bob/Fantômas, tombant un autre de ses masques, celui du Père Moche, de mobiliser la police pour prendre au piège Juve, emprisonné parce qu'on le prend pour Fantômas et libéré comme tel par la bande d'apaches que Fantômas commande et manipule! En bref, le lecteur s'enfonce dans un texte qui accumule les mystifications et méprises en matière d'identité(s), au point que Fantômas, véritable trou noir identitaire, peut être considéré comme une fantasmagorie, et donc à ce titre un écran à fantasmes. C'est ce que n'hésite d'ailleurs pas à déclarer JC Vareille :

«[...] il émane bien de ce que, faute d'un terme plus approprié, on appellera «l'inconscient». Il est «l'autre », le perpétuellement autre, celui que l'on ne peut saisir en dépit de sa présence hallucinante, celui qui grouille, fourmille et est toujours ailleurs, ici et au delà, présent et absent, celui qui agit ou fait agir et que l'on est incapable de discerner. Bien sûr, en tant que tel, il se rattache au principe de plaisir et se soucie peu des obstacles de la réalité.

[...] l'Insaisissable est celui qui détermine sans être déterminé, qui peut tout et sur lequel on ne peut rien, à la fois l'envers et le dedans, le double, l'autre de Juve et de Fandor qu'il obsède] et finalement de tous les lecteurs, le plus efficace des Premiers Rôles et des personnages le plus flou. $\gg 28$

Tel un emblème, par son succès foudroyant et durable de criminel « voleur de visage », Fantômas nous met du même coup sur la piste d'un processus décisif en matière d'identité perverse et de romanesque, qui ne tient plus à la seule thématisation par la fiction des détournements de l'identité et de ses déviances mais de la relation crime voir: Dominique Kalifa, L'Encre et le sang. Récits de crimes et société à la Belle Époque (Paris: Fayard, 1995). 28 Jean-Claude Vareille, L'Homme Masqué, le Justicier et le Détective..., pp. 142-143. 
foncièrement ambivalente que le lecteur construit avec les «mauvais genres » qui l'invitent à se délecter de la mise en scène de la violence. Comme l'avait noté Daniel Couégnas en forgeant le sixième critère de son «modèle paralittéraire », l'identification provoquée par la fiction populaire, lorsqu'elle évoque des relations dominant/dominé ou Méchant/Victime, peut en effet relever d'une «ambivalence sado-masochiste», ou encore s'avérer « jubilatoire, transgressive et iconoclaste » lorsque - précisément comme dans Fantômas - «dans les séquences de poursuite, la relation poursuivant/poursuivi est susceptible d'entraîner un basculement du processus identificatoire »29. Corollairement, et peut-être encore plus fondamentalement, c'est la production même du récit, en tant que machine textuelle à gratifications fantasmatiques pour la part du lecteur que Vincent Jouve propose, après Michel Picard, de nommer le « lu », qui présuppose un assentiment du lecteur sinon au «côté obscur de la force » du moins à sa perpétuelle renaissance, car « autoriser la perpétuation du Mal, c'est permettre que la série se poursuive » «La paralittérature exacerbe [donc d'après Couégnas] l'antagonisme du discours moralisateur et du plaisir de la lecture, qui sont néanmoins complices ! »30.

A faire défiler en accéléré quelques figures représentatives des criminels hantant le roman populaire français du XIXe siècle et de la Belle Epoque, nous aboutissons alors, en raisonnant sur «l'effet-personnage » sur le lecteur plutôt que de se limiter à une approche textualiste soulignant la stéréotypie des figures criminelles, à reconsidérer l'ambivalence même du plaisir procuré par la fiction et pris à la fiction. C'est sur ce dernier point que je voudrais conclure : la stimulation de l'identité perverse du lecteur par ce que je me suis risqué à appeler naguère le « storyplaying », i.e. l'investissement et la traversée du récit par le lecteur31. L'entêtante et souvent rayonnante présence de figures criminelles révèle en effet que la puissance consolatoire de la fiction romanesque, et particulièrement pour nombre de genres dits de grande consommation (polar, thriller, aventure, espionnage, horreur, porno ...) repose souvent sur un pacte d'écriture et « un contrat de lecture » placés sous le signe de la duplicité et de l'ambivalence, valeurs cardinales du storyplaying. Dans son essai de synthèse Matthieu Letourneux, en

\footnotetext{
29 Daniel Couégnas, Introduction à la Paralittérature..., p. 177.

30 Daniel Couégnas, Introduction à la Paralittérature..., pp. 177-178.

31 Jacques Migozzi, « Storyplaying. La machine à fabriquer ses histoires et à apaiser son esprit. », Finding the Plot, Diana Holmes, Dave Platten, Jacques Migozzi, Loïc Artiaga, dirs. (Cambridge: Cambridge Scholar Press, 2013), pp. $32-44$
} 
rappelant le double sens irréductible de toute catharsis, tout à la fois «processus de purgation (l'une des traductions du terme), d'élimination des passions par leur spectacle, [...][ et ] compréhension critique des passions, [...] travail de purification (autre traduction du terme). »32, a pu ainsi, en consonance avec les analyses présentées ici, souligner le «paradoxe central du roman d'aventures, qui joue à tous les niveaux du texte » et montrer que son succès tient à son ambivalence dans la mise en récit et en scène(s) de la violence transgressive :

«En un sens, le roman paraît condamner les valeurs de sauvagerie, à travers un discours moral, un dispositif actantiel manichéen, et une mise à distance de l'univers de l'aventure (dépaysement et esthétique du romanesque); à l'inverse, le cœur du texte consiste en la mise en scène de cet univers sauvage à travers une succession de mésaventures violentes, la figuration d'un espace de liberté à la fois cruel, sans lois et infiniment plus riche que l'espace réel du quotidien dans lequel le héros peut entièrement laisser s'exprimer son libre-arbitre. [...] Littérature d'évasion, le roman d'aventures privilégie l'écart avec le quotidien, un romanesque qui, comme la rêverie et le jeu, valorise le fantasme. Le récit met en scène un principe de plaisir triomphant dans un univers de fiction du possible, à la fois attirant et effrayant. $\gg 33$

Extrapolons hardiment: lorsque le lecteur s'encanaille avec de mauvais genres aux charmes équivoques, sa lecture cathartique ne lui fait-elle pas goûter aux délices de « la mauvaise foi » selon une autre formule de Letourneux ? Cette proposition fait écho en tous cas aux analyses de Jean-Marie-Schaeffer qui, lorsqu'il raisonne sur «le romanesque » et son « rôle important dans notre économie mentale »34, distingue deux grandes tendances, un romanesque blanc , optimiste ou heureux , et un romanesque noir, malheureux ou pessimiste, puis note leur fréquent mixage :

« On a souvent noté la tendance par exemple dans le roman grec à détailler les aventures des héros et à s'attarder sur les outrages qu'ils subissent. En fait cela montre qu'il est rare que le romanesque blanc lui-même se passe entièrement de toute incursion dans le romanesque noir» 35

32 Matthieu Letourneux, Le Roman d'aventure (Limoges: PULIM, 2010), p. 391.

33 Matthieu Letourneux, Le Roman d'aventure..., pp. 351-352.

34 Jean-Marie Schaeffer, «Le romanesque »<www.vox-poetica.org〉, p. 13.

35 Jean-Marie Schaeffer, « Le romanesque »..., p. 9. 
Et de poursuivre (et pour nous de conclure) : «notre méchanceté - qui n’est pas moins « humaine » que notre bonté - a elle aussi besoin de nourritures fictionnelles. »36 